\title{
Nebensächlich? Vorwort zum Nebentext
}

\author{
Lily Tonger-Erk $\cdot$ Niels Werber
}

Online publiziert: 18. Juli 2018

(C) Springer-Verlag GmbH Deutschland, ein Teil von Springer Nature 2018

Den in dieser Ausgabe der LiLi versammelten Beiträgen zum Thema »Hauptsache Nebentext! Regiebemerkungen im Drama $\ll^{1}$ liegt eine Versuchsanordnung zugrunde: Was bekommen wir in den Blick, wenn wir den Fokus der Dramenforschung ausnahmsweise vom Haupttext, dem Dialog, abwenden und stattdessen auf den Nebentext richten: auf jene Textabschnitte »vor, in, zwischen oder nach den direkten Reden im Drama «². Warum sich dieser Blickwechsel lohnt, warum er sogar notwendig ist, um der literarischen Gattung Drama gerecht zu werden, erscheint nicht auf den ersten Blick ersichtlich. Schließlich lässt bereits Roman Ingardens asymmetrisches Begriffspaar Haupttext/Nebentext vermuten, dass es sich hier um einen im Vergleich zum Dialog nebensächlichen Bestandteil des dramatischen Textes handelt. ${ }^{3}$ Holt man den Nebentext hingegen aus dem Schatten einer normativen literaturwissenschaftlichen Bestimmung, welche den Dialog als gattungskonstituierendes Merkmal des Dramas absolut setzt, ${ }^{4}$ erweist er sich als ein elementarer Bestandteil

\footnotetext{
1 Die Beiträge gehen zurück auf den Workshop »Hauptsache Nebentext! Regiebemerkungen im Drama«, der von Lily Tonger-Erk und Friederike Günther konzipiert wurde und vom 25. bis 26. November 2016 am Deutschen Seminar der Universität Tübingen stattgefunden hat.

2 Weimar, Klaus: »Regieanweisung«. In: Reallexikon der deutschen Literaturwissenschaft. Bd. 3. Hg. von Jan-Dirk Müller. Berlin 2007, S. 251-253, hier S. 251. Weimar schreibt zwar unter dem Lemma >Regieanweisung<, wechselt innerhalb des Artikels jedoch sogleich zur Definition des >Nebentextes $<$.

3 Vgl. Ingarden, Roman: Das literarische Kunstwerk. 2. verb. u. erw. Aufl. Tübingen 1960, S. 339.

4 Vgl. Szondi, Peter: Theorie des modernen Dramas (1880-1950). Frankfurt a.M. 1965.
}

L. Tonger-Erk $(\bowtie)$

Deutsches Seminar, Eberhard Karls Universität Tübingen, Tübingen, Deutschland

E-Mail: lily.tonger-erk@uni-tuebingen.de

N. Werber

Germanistisches Seminar, Universität Siegen, Siegen, Deutschland

E-Mail: werber@germanistik.uni-siegen.de 
des dramatischen Textes, der unverzichtbare rahmende, erzählende, kommentierende, strukturierende und illusionsbildende (oder auch -zerstörende) Funktionen im Drama übernimmt. ${ }^{5}$

Ingarden führt das Begriffspaar Haupttext/Nebentext im Jahr 1931 als maßgebliches Unterscheidungskriterium des Dramas im Vergleich mit anderen Gattungen so ein:

»Vor allem ist auffallend, daß in einem > geschriebenen< Drama zwei verschiedene Texte nebeneinander laufen: einerseits der Nebentext, d.h. die Angaben darüber, wo, in welcher Zeit usw. sich die betreffende Geschichte abspielt, wer gerade spricht und eventuell auch, was er momentan tut usw.; andererseits der Haupttext selbst. Der letztere besteht ausschließlich aus Sätzen, die von den dargestellten Personen > wirklich<ausgesprochen sind. Durch die Angabe der jeweiligen sprechenden Person gewinnen die zu dem Haupttext gehörenden Sätze gewissermaßen ein >Anführungszeichen $<. \ll^{6}$

Die beiden »Texte« des Dramas laufen nicht nur nebeneinander, wie Ingarden bemerkt, sondern wirken zusammen: Der Haupttext wird vom Nebentext »eingeschachtelt«, situiert und modifiziert. Der Dialog als »>wirklich < ausgesprochen [e]《 Figurenrede, der den Mittelpunkt aller auf die »Gegenwärtigkeit« und »Absolutheit « abonnierten Dramenkonzeptionen ${ }^{7}$ bietet, wird insofern durch den Nebentext allererst hervorgebracht. Daher ist der Nebentext einerseits elementarer Bestandteil des Dramas. Andererseits bleibe er eben »immer nur Nebentext« - die von Ingarden selbst vorgenommene Kursivierung macht sichtbar, dass die in der Begriffswahl transportierte Hierarchie programmatisch ist.

Für die Nebensächlichkeit des Nebentextes werden zwei Argumente angeführt. Erstens müsse sich allein aus dem Haupttext »die ganze zur Darstellung zu bringende Geschichte « ergeben, allein der Dialog sei also handlungstragend. ${ }^{8}$ Zweitens wird die Existenz des Nebentextes auf den schriftlichen Text beschränkt: »In einem Schauspiel fällt der Nebentext als Text fort. ${ }^{9}$ Die (Ir-)Relevanz des Nebentextes hängt also von einem handlungs- und theaterorientierten Gattungsbegriff ab. Diesen herkömmlichen Dramenbegriff stellen die Beiträge dieses Heftes grundsätzlich zur Disposition. ${ }^{10}$ Denn der normative Gattungsbegriff begründet die geringe Be-

\footnotetext{
5 Martin Ottmers nennt drei Funktionen von Nebentexten: Sie »arrangieren, situieren [und] kommentieren« die Figurenrede. Ottmers, Martin: »Drama«. In: Reallexikon der deutschen Literaturwissenschaft. Bd. 1. Hg. von Klaus Weimar u.a. Berlin/New York 1997, S. 392-396, hier S. 392. Zur Interferenz von Hauptund Nebentext vgl. Fischer-Lichte, Erika: »Der dramatische Dialog. Theater zwischen Schriftlichkeit und Mündlichkeit.« In: Wolfgang Raible (Hg.): Symbolische Formen, Medien, Identität. Jahrbuch $1989 / 90$ des Sonderforschungsbereichs »Übergänge und Spannungsfelder zwischen Mündlichkeit und Schriftlichkeit«. Tübingen 1991, S. 25-54, hier S. 32-37.

6 Ingarden, Roman: Das literarische Kunstwerk. 2. verb. u. erw. Aufl. Tübingen 1960, S. 220.

7 Vgl. Marx, Peter W.: »Dramentheorie«. In: Handbuch Drama. Theorie, Analyse, Geschichte. Hg. von Peter W. Marx. Stuttgart 2012, S. 1-11.

8 Ingarden, Roman: Das literarische Kunstwerk. 2. verb. u. erw. Aufl. Tübingen 1960, S. $221 \mathrm{f}$.

9 Ingarden, Roman: Das literarische Kunstwerk. 2. verb. u. erw. Aufl. Tübingen 1960, S. 339.

10 Vgl. in diesem Heft: Tonger-Erk, Lily: »Das Drama als intermedialer Text. Eine systematische Skizze zur Funktion des Nebentextes «.
} 
achtung des Nebentextes in der literaturwissenschaftlichen Forschung, die Szondis Dogma der »Absolutheit « des Dramas anhängt. ${ }^{11}$ Dagegen sieht sich die Theaterwissenschaft für die Analyse des Nebentextes nicht zuständig, fokussiert sie doch die plurimedialen Zeichen, in welche der Nebentext (wenn überhaupt) in der Aufführung transformiert wird.

Eine mögliche Nebentext-Forschung steht insofern zwischen den Stühlen: einer Literaturwissenschaft, welche die doppelte Medialität der Gattung Drama als Text und als Aufführung nicht selten zum Anlass nimmt, einen der Pole zu übersehen, und einer Theaterwissenschaft, die in der Bemühung um institutionelle Eigenständigkeit die Loslösung des Theaters vom dramatischen Text betreibt. Ausgehend von der These, dass dem Nebentext eine Schlüsselposition in Bezug auf die gattungsspezifischen, medialen und kommunikativen Implikationen des Dramas zukommt, plädieren die Beiträge dieses Heftes für eine Profilierung des Nebentextes als genuines Forschungsfeld der Literaturwissenschaft. Sie betrachten den Nebentext als konstitutiven Bestandteil des dramatischen Textes, der unabhängig von einer möglichen szenischen Darstellung existiert. Stellt man den Nebentext in den Fokus, ergeben sich viele Fragen, die in systematischer Hinsicht keineswegs geklärt und in historischer Hinsicht selten vergleichend beantwortet wurden: Wer spricht im Nebentext? An wen richtet sich der Nebentext? In welcher Relation steht er zum Haupttext? Wie gestaltet er das Verhältnis von Rede und Aktion? Welche (erzählende, kommentierende, strukturierende, situierende) Funktion kommt ihm zu? Wie vermittelt er zwischen Dramentext und (idealer, imaginärer, realer) Aufführung? Was verrät er über die zeitgenössische Theaterpraxis oder wie setzt er sich über diese hinweg? Und nicht zuletzt: Wie verändert sich seine Bedeutung und Funktion in der Geschichte des Dramas bis hin zur Postdramatik?

Am Beispiel von zwei Prinzessinnendramen Elfriede Jelineks, Der Tod und das Mädchen IV (Jackie) sowie Der Tod und das Mädchen V (Die Wand) aus dem Jahr $2002^{12}$ lassen sich die delikaten Implikationen dieser Fragen in aestheticis illustrieren. Dass postdramatische Theatertexte trotz ihres kritischen Umgangs mit der dramatischen Tradition den Nebentext nicht verabschieden, sondern im Gegenteil sein semantisches Potenzial ausloten und seine Funktionalität sowohl für die Aufführung als auch für die Lektüre erproben, ist bemerkenswert. Der Nebentext wird zum Ort dramatischer Selbstreflexion. In Der Tod und das Mädchen IV (Jackie) stellt der Nebentext ein Bewusstsein der Tatsache aus, dass seine Chancen, realiter >befolgt< zu werden, äußerst gering sind. Damit einher geht die Frage nach der Autorität des Autors bzw. der Autorin über die Aufführung, die nach herkömmlichem Verständnis den in sich »unvollständig[en] « Dramentext ja erst »vollendet «. ${ }^{13}$ Der Nebentext zu Beginn des vierten Prinzessinendramas ist denn auch nicht in dem seit dem

\footnotetext{
${ }^{11}$ Einen Überblick über die Forschung zum Nebentext bieten: Detken, Anke: Im Nebenraum des Textes. Regiebemerkungen in Dramen des 18. Jahrhunderts. Tübingen 2009, S. 1-40; Hauthal, Janine: Metadrama und Theatralität. Gattungs- und Medienreflexion in zeitgenössischen englischen Theatertexten. Trier 2009, S. 110-128. Vgl. auch den Beitrag von Lily Tonger-Erk in diesem Heft.

12 Jelinek, Elfriede: Der Tod und das Mädchen I-V. Prinzessinnendramen. Berlin 2003. Uraufführung von Teil IV und V: 24.11.2002 am Deutschen Theater Berlin (Regie: Hans Neuenfels).

13 Asmuth, Bernhard: Einführung in die Dramenanalyse, 7. aktual. u. erw. Aufl. Stuttgart/Weimar 2009, S. 10.
} 
18. Jahrhundert konventionalisierten Präsens ${ }^{14}$ formuliert, das - um nur ein klassisches Beispiel zu nennen - Schillers Wallensteins Tod, 1. Aufzug, prägt:

»Ein Zimmer, zu astrologischen Arbeiten eingerichtet und mit Sphären, Karten, Quadranten und anderm astronomischem Geräte versehen. Der Vorhang von einer Rotunde ist aufgezogen, in welcher die sieben Planetenbilder, jedes in einer Nische, seltsam beleuchtet, zu sehen sind. SENI beobachtet die Sterne, WALLENSTEIN steht vor einer großen, schwarzen Tafel, auf welcher der Planeten Aspekt gezeichnet ist. «15

An Stelle einer solchen ausgefeilten Beschreibung, die Bühnenbild und Choreographie buchstäblich präsentiert, erläutert in Jelineks Text eine Instanz in der ersten Person Singular tastend und vorsichtig im Konjunktiv, wie der Dramentext in der Aufführung umgesetzt werden »sollte« und »könnte«. Dabei suggeriert sie durch die fingierte Mündlichkeit eine direkte Kommunikationssituation zwischen Autorin und Theaterschaffenden:

»Jackie sollte in einem Chanel-Kostüm auftreten, denke ich (da müssen Sie aber schon sehr gute Gründe haben, wenn Sie das anders machen!). Man könnte auch als Vorbild dieses letzte Foto im Central Park (mit Maurice Tempelsman), das auf der Bank nehmen, Trenchcoat, Perücke (da Haare durch Chemo ausgegangen), Sonnenbrille und Hermès-Kopftuch.

Sie sollte in jedem Fall schwer arbeiten. Ich stelle mir vor, daß sie ihre ganzen Toten, die Kinder, na, der Embryo und die beiden toten Babies sind nicht so schwer, aber dafür die toten Männer, Jack, Bobby, Telis (»Ari«), das ergibt ein ganz hübsches Gewicht, was?!, also, wie soll ich sagen, diese Toten soll sie hinter sich herschleifen wie beim Tauziehen. Oder ein Wolgaschiffer sein Schiff. Das kann ich Ihnen nicht erleichtern. [...]

Aber Sie werden ja sicher was ganz andres machen. ${ }^{16}$

Dass hier die Autorin selbst spreche, dass es Elfriede Jelinek sei, die Vorschläge für die Inszenierung, Kostüme und Choreographie ihres Dramentextes auf der Bühne mache, erscheint zunächst naheliegend und ganz im Einklang mit der traditionellen Dramenforschung. Schon Ingarden nennt als Sprecher des Nebentextes den Autor: »In einem geschriebenen Drama gibt es [...] zwei verschiedene Texte: den Haupttext, d.h. die von den dargestellten Personen gesprochenen Worte und Sätze, und den Nebentext, d.h. die vom Autor gegebenen $>$ Informationen $<. \ll{ }^{17}$ Bis heute wird der Nebentext als unvermittelte Autorrede wahrgenommen. ${ }^{18}$ Während eine narrato-

\footnotetext{
14 Vgl. Detken, Anke: Im Nebenraum des Textes. Regiebemerkungen in Dramen des 18. Jahrhunderts. Tübingen 2009, S. 389.

15 Schiller, Friedrich: Wallensteins Tod. Tragödie in fünf Aufzügen, I. In: Schillers Werke. Nationalausgabe. Bd. 8. Hg. von Lieselotte Blumenthal. Weimar 1949, S. 177.

16 Jelinek, Elfriede: »Der Tod und das Mädchen IV (Jackie)«. In: Elfriede Jelinek: Der Tod und das Mädchen I-V. Prinzessinnendramen. Berlin 2003, S. 65-100, hier S. $65 f$.

17 Ingarden, Roman: Das literarische Kunstwerk. 2. verb. u. erw. Aufl. Tübingen 1960, S. 339 [Hervorhebungen von Ingarden].

18 Vgl. bspw. Zipfel, Frank: Fiktion, Fiktivität, Fiktionalität. Analysen zur Fiktion in der Literatur und zum Fiktionsbegriff in der Literaturwissenschaft. Berlin 2001, S. 310f. Einen detaillierten Überblick über die
} 
logisch fundierte Literaturwissenschaft gewohnt ist, die Instanz der Aussage und der Produktion eines epischen Textes zu unterscheiden, wird dem dramatischen Text ein vermittelndes Kommunikationssystem abgesprochen. ${ }^{19}$ Dagegen plädiert Alexander Weber im Rückgriff auf eine postklassische transgenerische Narratologie ${ }^{20}$ für die grundsätzliche Annahme einer »erzählende[n] bzw. vermittelnde[n] Instanz des Dramas«, die sich bereits durch Selektion, Arrangement und Stilisierung des Dialogs zeigt. ${ }^{21}$ Dass sich diese Instanz hier als Autorin im Kampf um die Deutungshoheit über das Drama stilisiert, ist für einen Nebentext historisch eher die Ausnahme als die Regel: »Paradoxerweise markieren gerade instruktive Formulierungen einen unkonventionellen Gebrauch des Nebentexts, da sie einen Hinweis auf die fiktionale Qualität der als Instruktion gelesenen Assertion beinhalten und entgegen der Rezeptionsillusion der Unvermitteltheit des (dramatischen) Theatertexts im Zuge der Lektüre auf dessen >theatrale< Vermittlung bzw. Vertextung aufmerksam machen $\ll^{22}$, bemerkt Janine Hauthal. Die ostentative instruktive Formulierung in Jelineks Nebentext verweist also auf die Medialität des Dramentextes, während die ausgestellte Metaphorizität (der Vergleich der Choreographie mit einem »Tauziehen«bzw. einem »Wolgaschiffer«) darauf hinweist, dass der Nebentext nicht in der Vermittlung von technischen Informationen aufgeht, sondern als Teil eines »literarischen Kunstwerks « wahrgenommen werden will. ${ }^{23}$

Neben der Frage, wer im Nebentext spricht, wirft Der Tod und das Mädchen IV (Jackie) auch diejenige auf, wen der Nebentext adressiert. Während Schillers konventionell formulierter Nebentext eine direkte Anrede vermeidet, jedoch implizit von einer im Zuschauerraum platzierten Rezeptionsinstanz ausgeht, ${ }^{24}$ spricht Jelineks Nebentext direkt seine Adressaten an. Ob sich die formelle Anrede »Sie« an

Positionierung der Forschung zur Frage, wer den Nebentext spricht, bietet Hauthal, Janine: Metadrama und Theatralität. Gattungs- und Medienreflexion in zeitgenössischen englischen Theatertexten. Trier 2009, S. 110-128.

19 Vgl. Hamburger, Käte: Die Logik der Dichtung. Stuttgart 1957, S. 118; Pfister, Manfred: Das Drama. 11. Aufl. Stuttgart 2001, S. 20-22.

20 Vgl. Tschauder, Gerhard: »Wer >erzählt< das Drama? Versuch einer Typologie des Nebentexts« In: Sprache und Literatur in Wissenschaft und Unterricht 68 (1991), S. 50-67. Jahn, Manfred: »Narrative Voice and Agency in Drama. Aspects of a Narratology of Drama«. In: New Literary History 32 (2001), S. 659-679. Wolf, Werner: »Der Prolog als traditionelle Form dramatischer Anfangsrahmung: Probleme der Definition und der Funktionsgeschichte im englischen Drama des 19. Jahrhunderts «. In: Hugo Keiper/ Maria Löschnigg/Doris Mader (Hg.): Metamorphosen. Englische Literatur und die Tradition. Heidelberg 2006, S. 203-238. Muny, Eike: Erzählperspektive im Drama. Ein Beitrag zur transgenerischen Narratologie. München 2008.

21 Weber, Alexander: Episierung im Drama. Ein Beitrag zur transgenerischen Narratologie. Berlin/Boston 2017, S. 159-163. Weber argumentiert im Rückgriff auf: Jahn, Manfred: »Narrative Voice and Agency in Drama. Aspects of a Narratology of Drama«. In: New Literary History 32 (2001), S. 659-679.

22 Hauthal, Janine: Metadrama und Theatralität. Gattungs- und Medienreflexion in zeitgenössischen englischen Theatertexten. Trier 2009, S. 128.

23 Vgl. Poschmann, Gerda: Der nicht mehr dramatische Theatertext. Aktuelle Bühnenstücke und ihre dramaturgische Analyse. Tübingen 1997, S. 329.

24 Klaus Weimar stellt fest, dass Regiebemerkungen grundsätzlich »aus der Sicht des Publikums bei einer theatralen Aufführung formuliert werden (z.B. kommt von rechts)«. Weimar, Klaus: »Regieanweisung «. In: Reallexikon der deutschen Literaturwissenschaft. Bd. 3. Hg. von Jan-Dirk Müller. Berlin 2007, S. 251-253, hier S. 251. 
einen einzelnen Regisseur bzw. eine Regisseurin oder an das Kollektiv aller an der Inszenierung Beteiligten (Schauspieler/innen, Kostüm- und Bühnenbildner/innen etc.) wendet, ist dabei sekundär. Medial bedeutsam erscheint hingegen, dass der Nebentext in seiner Funktion als Regiebemerkung eher Theaterschaffende zu adressieren scheint als Leser bzw. Leserinnen. Als autoritäre Regieanweisung ist dieser vorsichtig im Konjunktiv formulierte Nebentext dennoch nicht zu bezeichnen, eher als dialogisches Aufzeigen imaginärer Regiemöglichkeiten (»ich stelle mir vor«). Und diese Möglichkeiten eröffnen sich ebenso den Lesern und Leserinnen als imaginäre Regisseure und Regisseurinnen. Denn so überzeugt, wie das Ich vor dem Hintergrund zeitgenössischer Autortheorie und Regietheaterpraxis von der Aussichtslosigkeit einer szenischen Umsetzung seiner Bühnenanweisungen erscheint, müsste es eigentlich auf den Nebentext gänzlich verzichten: »Aber sie werden ja sicher was ganz andres machen. « Dass dem nicht so ist, hebt die Bedeutung des Nebentextes für die Lektüre des Dramentextes hervor, für eine imaginäre Inszenierung >im Kopf< des Lesers bzw. der Leserin.

Nicht zuletzt reflektiert der Nebentext also die zentrale Frage nach dem Verhältnis zwischen Dramentext und Aufführung. Die Theaterwissenschaft hat seit ihrer disziplinären Begründung durch Max Herrmann darum gekämpft, die szenische Darstellung von der Dominanz des dramatischen Textes zu lösen und als eigenständige künstlerische Darstellungsweise zu profilieren. Damit kann der dramatische Text nicht länger als konstitutiver Bestandteil der szenischen Darstellung gelten,

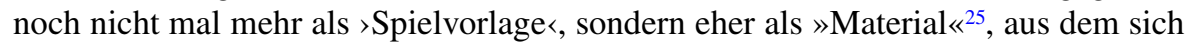
die Aufführung bedient. Daraus folgt, dass der Dramen-Autor bzw. die -Autorin die Textherrschaft über die Aufführung verliert, während der Regisseur bzw. die Regisseurin eine Aufwertung erfährt. Die Konjunktur des Regie-Theaters in Deutschland feuert diesen »struggle for interpretive control of the production $\ll^{26}$ noch an. Zum expliziten Austragungsort eines solchen Machtkampfs wird der Nebentext des letzten Prinzessinnendramas, Der Tod und das Mädchen V(Die Wand):

»Diesmal allerdings, Herr Chef, Frau Chefin, müssen Sie, zumindest in den Grundzügen, die szenischen Anweisungen für die Bühnenaktionen ausführen, die ich vorgegeben habe, denn diesmal sind sie Teil des Textes. Tut mir echt leid. $\ll^{27}$

»Herr Chef, Frau Chefin« - ähnlich ironisch adressieren schon Nebentexte in Jelineks frühen Stücken wie Krankheit oder Moderne Frauen (1987) den Regisseur als »Herr Vorsitzender $\aleph^{28}$ - und formulieren damit eine gespielte Unterwerfung. Indem der Nebentext - ausnahmsweise! - als »Teil des Textes« proklamiert wird, wird die zugrunde liegende traditionelle Dichotomie offenbar: Einerseits scheint der

\footnotetext{
25 Vgl. Fischer-Lichte, Erika: Theaterwissenschaft. Eine Einführung in die Grundlagen des Fachs. Tübingen 2010, S. 93-100.

26 Carlson, Marvin: »The Status of Stage Directions«. In: Studies in the Literary Imagination 24/2 (1991), S. 37-48, hier S. 46.

27 Jelinek, Elfriede, »Der Tod und das Mädchen V (Die Wand)«. In: Elfriede Jelinek: Der Tod und das Mädchen I-V. Prinzessinnendramen. Berlin 2003, S. 103-143, S. 103.

28 Jelinek, Elfriede: Krankheit oder Moderne Frauen. Köln 1987, S. 9.
} 
Haupttext zum Text und dem Autor zu gehören, andererseits fällt der Nebentext in den Hoheitsbereich der Aufführung und damit des Regisseurs. Da der Regisseur kaum noch an den Text und schon gar nicht an den Nebentext gebunden ist, ${ }^{29}$ wie Jelineks Nebentexte selbst ausstellen, wird dem Nebentext eine Relevanz für die Inszenierung doppelt abgesprochen. Er ist »für die Bühnenpraxis so gut wie entbehrlich «. ${ }^{30}$ Umso deutlicher ist auf die Funktion der Imaginationslenkung für die Leserin hinzuweisen, die über die Lektüre aufgefordert wird, sich »Bühnenaktionen « vorzustellen.

So ist es kein Zufall, dass nahezu alle Beiträge in diesem Heft vehement für eine $>$ Rehabilitierung< des Dramas als eigenständigem literarischen Text eintreten. ${ }^{31}$ Sie gehen gegen eine herkömmliche Dramenforschung an, wie sie noch in jüngeren literaturwissenschaftlichen Lehrwerken verbreitet wird, die problematisch sind, »nicht nur weil sie den Respekt für die Selbstständigkeit des Mediums Theater vermissen lassen, sondern auch weil sie de facto die Selbstständigkeit des Textes in Frage stellen $\ll^{32}$ - wie Georg-Michael Schulz 2003 bemerkt. Diese Forschung sieht das Drama erst in der Aufführung verwirklicht, ordnet den Nebentext der Aufführung unter und ignoriert seine Funktion für die Lektüre. Prominent zurückverfolgen lässt sich eine solche Ansicht auf Julius Petersen, der 1904 schreibt, in den »Bühnenanweisungen vollzieht sich die - konstruierte - Metamorphose vom Epos zum Drama; vollendet ist sie erst mit der Aufführung. ${ }^{33}$ Doch bereits Petersen bemerkt, dass der Nebentext nicht in der Aufführung aufgeht:

»Da also die Bühnenanweisungen gewissermaßen einen Wechsel vorstellen, der erst bei der Aufführung eingelöst wird, müsste es ihre Grundeigenschaft sein, in der Darstellung restlos aufzugehen. Ganz gelingt es indessen der Aufführung nicht, alles hindurchzusieben; denn die Bühne kann wohl einen Saal darstellen, aber sie kann uns nicht beweisen, dass wir im Hause des Fiesko sind. $\ll^{34}$

Anstatt diese Beobachtung ernst zu nehmen, kritisiert Petersen jedoch solche >überschüssigen< Nebentexte als »novellistische[n] Rest«, mithin als fehlerhafte Gattungsverunreinigung. Petersen wertet die »Angaben [des Dichters], die man mit dem

\footnotetext{
${ }^{29}$ Vgl. Pavis, Patrice: »Die Inszenierung zwischen Text und Aufführung«. In: Zeitschrift für Semiotik 11.1 (1989), S. 13-27, hier S. 17.

${ }^{30}$ Bayerdörfer, Hans-Peter: »Regie und Interpretation oder Bühne und Drama. Fußnoten zu einem unerschöpflichen Thema«. In: Helmut Kreutzer/Dieter Zerlin (Hg.): Literatur - Theater - Museum. München 1987, S. 118-143, hier S. 130.

${ }^{31}$ Eine solche Rehabilitierung fordern und begründen ebenfalls: Jahn, Manfred: »Narrative Voice and Agency in Drama. Aspects of a Narratology of Drama «. In: New Literary History 32 (2001), S. 659-679, hier S. 662; Weber, Alexander: Episierung im Drama. Ein Beitrag zur transgenerischen Narratologie. Berlin/Boston 2017, S. 29.

32 Schulz, Georg-Michael: »Text und Inszenierung als Verwirrspiel. Der Komplex >Drama und Theater in jüngeren literaturwissenschaftlichen Einführungen «. In: Der Deutschunterricht 1/2003, S. 90-95, hier S. 93.

${ }^{33}$ Petersen, Julius: Schiller und die Bühne. Ein Beitrag zur Litteratur- und Theatergeschichte der klassischen Zeit. Berlin 1904, S. 5.

${ }^{34}$ Petersen, Julius: Schiller und die Bühne. Ein Beitrag zur Litteratur- und Theatergeschichte der klassischen Zeit. Berlin 1904, S. 6.
} 
unsinnigen Namen epische Bühnenanweisung bezeichnen könnte«, als Kinderkrankheiten von Jugenddramen ab, die von der unausgereiften Theaterschulung ihres Autors zeugen. ${ }^{35}$ So verstellt die normative Auffassung des Dramas als Spielvorlage und die Fokussierung auf die szenische Darstellung notwendig den Blick auf den Nebentext. Dagegen verweisen die Beiträge in diesem Heft auf die Medialität und Literarizität des Nebentextes.

Die systematischen Fragen, die sich aus der Fokussierung des Nebentextes ergeben, lassen sich - dies sei vorweggenommen - nur historisch konkret an individuellen Texten beantworten. Sie können die Analyse von Dramentexten schärfen, jedoch keine überhistorischen Generalisierungen zeitigen. So verstehen sich die folgenden Beiträge auch als systematisch orientierte Erkundungsgänge in die Geschichte des Nebentextes. Sie erfassen von der Frühen Neuzeit bis zur Postdramatik eine weite historische Spanne. Der Blick in die Geschichte zeigt: Die Antike kennt noch gar keine Regiebemerkungen, der Bühnenbezug ist als impliziter Nebentext gegeben. Das mittelalterliche geistliche Spiel enthält schon neben Sprechernamen auch einzelne anschauliche Beschreibungen, die auf die Bühne, Kostüme, Requisiten sowie die Aktion verweisen. ${ }^{36}$ Die Annahme, dass Regiebemerkungen in der Frühen Neuzeit noch nicht bemerkenswert seien, hält sich hartnäckig - wie in der derzeit aktuellsten Drameneinführung: »Das barocke Trauerspiel etwa weist nur in absoluten Ausnahmefällen knappste, dann in Fußnoten zum jeweiligen Vers gesetzte Hinweise etwa auf ein Hinknien oder Aufstehen einer Figur. Ansonsten sind die Texte regiebemerkungsfrei [...].« ${ }^{37}$ Dagegen zeigt Constanze Baum in diesem Heft an Dramen von Ayrer, Gryphius, Hallmann und Weise beispielhaft, dass bereits in allen Dramengattungen der frühen Neuzeit Nebentexte zu finden sind, die sich nicht nur auf eine Rekonstruktion der historischen Bühnenpraxis hin lesen lassen, sondern eine bedeutsame Funktion für die Geschehensvermittlung übernehmen. ${ }^{38}$ Die frühneuzeitlichen Dramentexte weisen eine druckgraphische Variantenvielfalt auf, die in der Skalierung sogar den Nebentext über den Haupttext triumphieren lassen kann. In der ersten Hälfte des 18. Jahrhunderts lässt sich dann eine fortschreitende Entwicklung von einem impliziten zum expliziten Anmerkungssystem beobachten, wie Anke Detken minutiös in der einzigen germanistischen Monographie zum Nebentext gezeigt hat, die innerhalb der letzten fünfzig Jahre erschienen ist. ${ }^{39}$ Nun entwickeln

\footnotetext{
35 So kritisiert und korrigiert Petersen eine Bühnenanweisung in Schillers Kabale und Liebe: »er eilt schnell fort und rennt - gegen den Präsidenten « (II,6). Da sich der Bindestrich nicht im rasanten Spiel umsetzen ließe, sieht er sich genötigt, eine »korrekte Bühnenanweisung « zum Vergleich anzubieten. Dazu wählt Petersen eine Regiebemerkung in Die Verschwörung des Fiesko zu Genua: »Giannettino will hinaus und stösst auf Fiesko. GIANETTINO zurückfahrend. Ha!« (III,9-10) Allein die Werkchronologie spricht in diesem direkten Vergleich - wie Petersen sicherlich weiß - gegen die These von einem Zusammenhang der theaterpraktischen Funktionalität und der Theatererfahrenheit des Autors. Vgl. Petersen, Julius: Schiller und die Bühne. Ein Beitrag zur Litteratur- und Theatergeschichte der klassischen Zeit. Berlin 1904, S. 7-8.

36 Vgl. Steiner, Jacob: Die Bühnenanweisung. Göttingen 1969.

37 Jeßing, Benedikt: Dramenanalyse. Eine Einfiuhrung. Berlin 2015, S. 31.

38 Vgl. in diesem Heft: Baum, Constanze: »Nebentexte als Bedeutungsträger im Drama der Frühen Neuzeit «.

39 Vgl. Detken, Anke: Im Nebenraum des Textes. Regiebemerkungen in Dramen des 18. Jahrhunderts. Tübingen 2009.
}

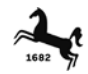


sich die uns heute noch geläufigen syntaktischen Konventionen des Nebentextes wie z.B. konventionelle Auf- und Abtrittsformeln und die Beibehaltung des Präsens. Mit dem Anstieg des Dramendrucks korrespondiert auch die wachsende Konventionalisierung des Nebentextes als Teil eines dramenspezifischen typographischen Dispositivs, wie Thomas Boyken in der vorliegenden Ausgabe an Kleists Lustspiel Der zerbrochne Krug herausarbeitet. ${ }^{40}$ Boyken entwickelt dabei die strukturierende Funktion des Nebentextes im Lustspiel. Im Sturm und Drang lässt sich dann eine massive Konjunktur der Regiebemerkungen und mithin eine Ausweitung ihres Funktionsspektrums beobachten - bemerkenswerterweise bei gleichzeitigem Mangel an Aufführungspraxis. Im Naturalismus überholt der Nebentext manches Mal quantitativ den Haupttext. Bertolt Brechts episches Theater wertet den Nebentext deutlich auf und funktionalisiert ihn als transgenerischen Beziehungsstifter, wie Irmtraud Hnilica in diesem Heft zeigt. ${ }^{41}$ Schließlich setzen Stücke wie Samuel Becketts Spiel ohne Worte (1 und 2) (1956) oder Peter Handkes Das Mündel will Vormund sein (1968) den Nebentext gar absolut und verzichten auf einen Haupttext. Dass und wie zuletzt postdramatische Theatertexte den Nebentext keineswegs ausschalten, sondern experimentell erproben, beobachten die Beiträge von Thomas Wortmann, Anke Detken und Janine Hauthal. ${ }^{42}$ Wortmann untersucht anhand von Christoph Schlingensiefs Theatertext Rosebud den Sonderfall eines Nebentextes, der nicht präskriptiv funktioniert, sondern vielmehr rückwirkend die (missglückte) Uraufführung protokolliert, kommentiert und idealisiert. Detken führt vor, wie Personenverzeichnisse, Sprecherbezeichnungen und Regiebemerkungen ihren ästhetischen Eigenwert in Texten von Heiner Müller, Fritz Kater und Dea Loher behaupten - dem vermeintlichen Fokus der Postdramatik auf die Aufführung zum Trotz. Und Hauthal zeigt, wie die Reduzierung des Nebentextes bzw. die Verschmelzung von Haupt- und Nebentext in Theatertexten von Martin Crimp, Mark Ravenhill und Simon Stephens Konventionen der dramatischen Darstellung unter- und zum Erzählen überlaufen.

\footnotetext{
40 Vgl. in diesem Heft: Boyken, Thomas: »Der Nebentext eines Lesedramas: Sprecherbezeichnung und Komik in Kleists Der zerbrochne Krug«.

41 Vgl. in diesem Heft: Hnilica, Irmtraud: »Zum Titularium in Brechts Mutter Courage und ihre Kinder: Plädoyer für einen starken Nebentextbegriff «.

42 Vgl. in diesem Heft: Wortmann, Thomas: »Haupttext, Nebentext - Klartext? Intermedialität und Autorität in Christoph Schlingensiefs Theaterstück Rosebud - Das Original (2002)«; Detken, Anke: »Kursiv Geschriebenes und KAPITÄLCHEN - Typologische Überlegungen zu Regiebemerkungen und Sprecherbezeichnungen in postdramatischen Theatertexten«; Hauthal, Janine: »Towards a Narrative Aesthetic? The Scarcity of Stage Directions as (Text-)Theatrical Challenge in Plays by Crimp, Ravenhill and Stephens «.
} 